\title{
Spectrum Utility: a Novel Metric for Efficient Spectrum Usage in Next-generation Networks
}

\author{
F. Bouali, K. Moessner \\ 5G Innovation Centre (5GIC) \\ University of Surrey, UK \\ Email: \{f.bouali, k.moessner\}@ surrey.ac.uk
}

\author{
M. Fitch \\ BT Research \\ Adastral Park, Ipswich IP5 3RE, UK \\ Email: michael.fitch@bt.com
}

\begin{abstract}
This paper proposes a novel spectrum utility (SU) metric that assesses the efficiency of spectrum usage by a set of heterogeneous applications. Unlike the traditional spectrum efficiency (SE), the proposed metric does not blindly consider the achievable bit-rate, but captures the most relevant performance metrics for each of the considered applications. Specifically, it is formulated as an aggregated utility that combines the satisfaction level with respect to the various requirements with an innovative pricing model based on it to derive the total revenue generated for the spectrum owner. To get insight into the usefulness of the proposed metric, the proposed methodology is instantiated for an illustrative use case, where a mixture of delay-sensitive (i.e., interactive video) and -tolerant (i.e., file transfer) applications are established in dense indoor deployments. The obtained results reveal that the proposed SU significantly outperforms the legacy $\mathrm{SE}$ in assessing how efficiently a limited frequency spectrum is utilised from the perspective of the total revenue, particularly when the quality-of-experience (QoE) perceived during video sessions is degraded. This calls for a novel $\mathrm{SU}$-aware ecosystem, where the spectrum sharing models, billing policies and resource allocation mechanisms (e.g., medium access control (MAC) and radio resource management $(\mathrm{RRM}))$ are jointly revisited to maximise the overall SU.
\end{abstract}

\section{Context/Motivation}

To assess the efficiency of spectrum usage by a given communication system, the spectrum efficiency (SE) metric has been very popular in both academia and industry to assess the achievable bit-rate in bits per second (bps) per Hertz of spectrum.

Since its early introduction in cellular networks [1], it has evolved considerably to meet various needs. In particular, it was extended in [2] into the area spectrum efficiency (ASE) expressed in bps/Hz/unit of area to capture the gain that may be introduced by frequency reuse in a given deployment area. The second major improvement is its redefinition based on the user-perceived throughput as a random variable whose statistics (e.g., cumulative distribution function (CDF)) could better characterise its variability due to the radio and load conditions. The latter approach is currently recommended by the third generation partnership project (3GPP) as reflected by the system performance metrics recently selected in Section A.2.1.4 of [3].

All these extensions, albeit useful, focus on the bit-rate, which is a valid performance metric for traditional data applications (e.g., file transfer), but cannot capture the more complex and heterogenous requirements associated with the wide range of emerging applications (e.g., high-quality video streaming and machine type communications).

To tackle this limitation, this paper proposes a novel spectrum utility (SU) metric to assess the efficiency of utilising a limited frequency spectrum by a set of heterogeneous applications. The proposed metric combines the satisfaction level achieved with respect to the various requirements with an innovative pricing model based on it to derive the total revenue generated for the spectrum owner (e.g., operator). From a practical standpoint, it could be considered as the return-oninvestment (ROI) when the acquired spectrum is exploited in a particular way e.g., by some radio access technologies (RATs) and/or to establish a particular set of applications.

Therefore, the first contribution of this paper is the development of a novel SU that assesses how efficiently a limited frequency spectrum is utilised from the perspective of the total revenue generated by the considered applications. The second contribution is the instantiation of the proposed metric for an illustrative use case, where a mixture of delay-sensitive (i.e., interactive video) and -tolerant (i.e., file transfer) applications are established in dense indoor deployments. Consequently, the third contribution is the benchmarking of the proposed SU against the traditional SE from both the total revenue and individual application perspectives.

The remainder of this paper is organized as follows. The system model is described in Section II including a review of the traditional SE approach. A novel SU metric is developed in Section III to assess the efficiency of spectrum usage by a set of heterogeneous applications. The proposed metric is instantiated in Section IV for an illustrative use case, where a mixture of delay-sensitive and -tolerant applications are established in dense indoor deployments. The obtained results are presented in Section $\mathrm{V}$, including the evaluation of the proposed metric together with the individual application performances. The conclusions and future directions are provided in Section VI.

\section{SySTEM MODEL}

A given deployment area $A\left[m^{2}\right]$ is considered, where $K$ available RATs $\left(\left\{R A T_{k}\right\}_{1 \leq k \leq K}\right)$ are available on a given spectrum bandwidth $W[H z]$. The various RATs are exploited by $N$ active users to establish a set of $M$ applications ( $\left.\left\{A_{m}\right\}_{1 \leq m \leq M}\right)$, each characterised by a set of $R_{m}$ requirements $\left(\left\{R e q_{m}^{r}\right\}_{1 \leq r \leq R_{m}}\right)$. For each application $A_{m}, N_{m}$ denotes the number of active users such that $\sum_{m=1}^{M} N_{m}=N$.

\section{A. Link spectrum efficiency}

For each $n$-th user of a given $m$-th application, the SE achieved at a given time $t$ when using $R A T_{k}$ is expressed in bps per Hertz of spectrum as:

$$
S E_{m, n, k}(t)=\frac{B_{m, n, k}(t)}{W}
$$

where $B_{m, n, k}(t)$ denotes the achievable bit-rate at time $t$. 


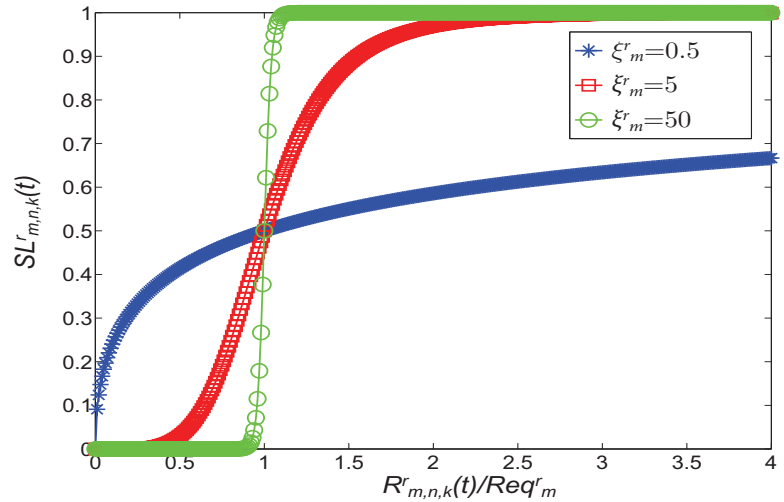

Fig. 1. Behavior of the marginal satisfaction level

\section{B. Area spectrum efficiency}

To capture the potential frequency reuse gain, the ASE is calculated as the aggregated link spectrum efficiency normalized to the deployment area:

$$
\begin{aligned}
A S E(t) & =\frac{1}{A} \sum_{m=1}^{M} \sum_{n=1}^{N_{m}} \sum_{k=1}^{K} S E_{m, n, k}(t) \\
& =\frac{1}{A \cdot W} \sum_{m=1}^{M} \sum_{n=1}^{N_{m}} \sum_{k=1}^{K} B_{m, n, k}(t)
\end{aligned}
$$

Both of the aforementioned metrics (i.e., $S E_{m, n, k}$ and ASE) focus on the achievable bit-rate and assume that every successfully transmitted bit contributes equally to the overall SE. This assumption is valid for a traditional file transfer, but may not hold for many emerging applications. For instance, few Kbits received within a given delay budget would be highly valuable for low-end sensors, while even few Mbits may not sustain a good quality-of-experience (QoE) for $4 \mathrm{~K}$ video streaming.

To overcome these limitations, a novel metric is developed is the next section.

\section{SPECTRUM UtILITY}

This section devises a novel metric to assess the efficiency of spectrum usage from the perspective of the total revenue generated by the considered applications.

\section{A. Application satisfaction levels}

This section proposes a generic formulation to evaluate the satisfaction level achieved by each of the considered applications.

For each $n$-th user of a given $m$-th application, the satisfaction level achieved at a given time $t$ when using $R A T_{k}$ is defined as:

$$
S L_{m, n, k}(t)=\prod_{r=1}^{R_{m}}\left(S L_{m, n, k}^{r}(t)\right)^{\alpha_{m}^{r}}, \quad \alpha_{m}^{r}>1 \forall r \in\left\{1, \ldots, R_{m}\right\} ;
$$

where $S L_{m, n, k}^{r}(t)$ denotes the marginal satisfaction level with respect to the $r$-th requirement (i.e., $R e q_{m}^{r}$ ). The controlling parameters $\left\{\alpha_{m}^{r}\right\}_{1 \leq r \leq R_{m}}$ could be adjusted differently to reflect the relative importance of the various requirements.

More specifically, the following formulation [4] is considered to assess the marginal satisfaction levels:

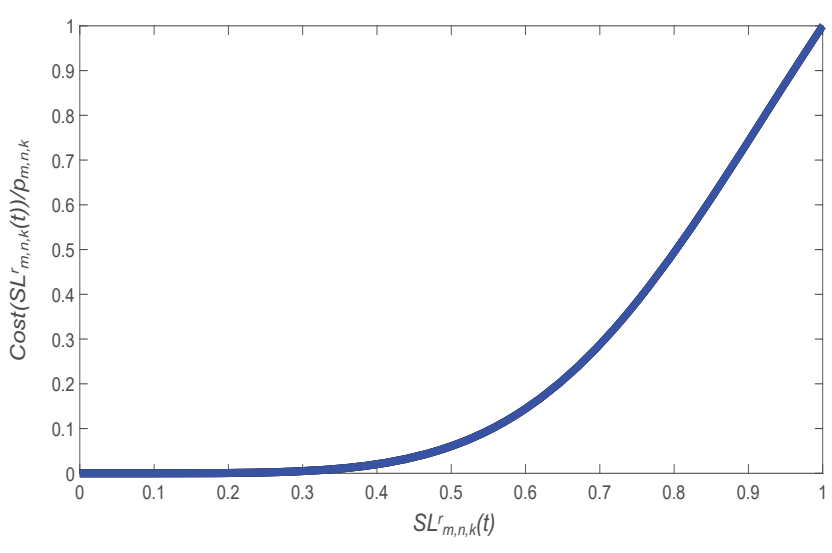

Fig. 2. Behavior of the considered pricing model, $\beta=5$

$$
S L_{m, n, k}^{r}(t)=\frac{\left(\frac{R_{m, n, k}^{r}(t)}{R e q_{m}^{r}}\right)^{\xi_{m}^{r}}}{1+\left(\frac{R_{m, n, k}^{r}(t)}{R e q_{m}^{r}}\right)^{\xi_{m}^{r}}}
$$

where $R_{m, n, k}^{r}(t)$ denotes the achievable performance with respect to Re $q_{m}^{r}$ when using $R A T_{k}$ and $\xi_{m}^{r}$ is a shaping parameter that allows the function to capture different degrees of elasticity with respect to $R e q_{m}^{r}$.

The proposed formulation belongs to the family of sigmoid functions [4]. To better analyse its behavior, Fig. 1 plots it as a function of the ratio $R_{m, n, k}^{r}(t) / R e q_{m}^{r}$ for different values of the shaping parameter $\xi_{m}^{r}$. It can be seen that $S L_{m, n, k}(t)$ is a monotonic increasing function of the achievable performance $R_{m, n, k}^{r}(t)$ that equals 0.5 at $R_{m, n, k}^{r}(t)=R e q_{m}^{r}$, and tends asymptotically to 1 . The marginal increase of the utility function for large performances $R_{m, n, k}^{r}(t)$ well above the requirement $R e q_{m}^{r}$ becomes progressively smaller especially when intermediate values of $\xi_{m}^{r}$ are used (e.g., $\xi_{m}^{r}=5$ ). Therefore, $S L_{m, n, k}(t)$ provides a measure of the satisfaction level with respect to the requirement $R e q_{m}^{r}$, with values ranging from 0 (low satisfaction) to 1 (high satisfaction).

\section{B. Overall spectrum utility}

For each $n$-th user of a given $m$-th application, the contribution to the overall $\mathrm{SU}$ is defined as the generated revenue per Hertz of spectrum:

$$
S U_{m, n, k}(t)=\frac{1}{W} \cdot\left(S L_{m, n, k}(t) \cdot \operatorname{Cost}\left(S L_{m, n, k}(t)\right)\right)
$$

where Cost(.) reflects the pricing policy i.e., price (or equivalently the willingness) to pay per unit of satisfaction level.

Without loss of generality, the following pricing model is considered:

$$
\operatorname{Cost}\left(S L_{m, n, k}(t)\right)=p_{m, n, k} \cdot\left(\frac{2 \cdot\left(S L_{m, n, k}(t)\right)^{\beta}}{1+\left(S L_{m, n, k}(t)\right)^{\beta}}\right)
$$

where $p_{m, n, k}$ is the maximum price that could be set differently for each application depending on the associated business model. An example of such tuning could be found in Section V-A.

As illustrated in Fig. 2, the considered formulation is a monotonically increasing function meaning that the price to pay is higher when the user is more satisfied, reaching its maximum (i.e., $p_{m, n, k}$ ) at full satisfaction (i.e., $S L_{m, n, k}(t)=1$ ). 
Note that the proposed pricing model would require to redefine the user subscription profiles and billing policy based on the application satisfaction levels, which would be a future-proof step to support the requirements of any emerging application.

Next, the area spectrum utility (ASU) is defined as the aggregated spectrum utility per unit of area:

$$
\begin{aligned}
A S U(t) & =\frac{1}{A} \sum_{m=1}^{M} \sum_{n=1}^{N_{m}} \sum_{k=1}^{K} S U_{m, n, k}(t) \\
& =\frac{1}{A \cdot W} \sum_{m=1}^{M} \sum_{n=1}^{N_{m}} \sum_{k=1}^{K} S L_{m, n, k}(t) \cdot \operatorname{Cost}\left(S L_{m, n, k}(t)\right)
\end{aligned}
$$

The proposed ASU provides a generalisation of the legacy ASE. As a matter of fact, it can be seen that (2) represents a particular case of (7) when $S L_{m, n, k}(t)=B_{m, n, k}(t)$ and $\operatorname{Cost}\left(S L_{m, n, k}(t)\right)=1$.

In what follows, the time argument will be dropped from the notation for the sake of simplicity.

\section{Use CASE: Delay-SEnSitive AND -TOLERANT APPLICATIONS in DENSE Indoor DePLOYMENTS}

\section{A. Considered environment}

- To model ultra-dense deployments, a single LTE macro cell (i.e., $K=1$ ) overlaid by a set of buildings is considered. Each building is structured according to the dualstripe layout [5] i.e., as two stripes of rooms with a corridor in-between, which corresponds in practice to e.g., the set of stores inside a shopping mall. The various propagation losses (i.e., indoor-indoor, outdoor-outdoor, indoor-to-outdoor and vice versa) are modeled using the hybrid building model that combines several well known propagation loss models [6].

- A set of LTE small cells are dropped randomly inside each building. As an illustrative example, Fig. 3 describes the signal-to-interference-and-noise-ratio (SINR) map obtained when a building composed of two 20-room stripes is considered with one small-cell placed in each room.

- The full list of LTE physical parameters is that of the outdoor scenario described in the Annex A of [7].

\section{B. Traffic mixture}

The set of $N$ users are randomly dropped inside the considered environment. Each user may establish one of the following $M=2$ applications:

- Interactive (i.e., delay-sensitive) video whose requirements are characterized in terms of a minimum bit-rate $B_{1}^{\min }$ and maximum end-to-end delay of $D_{1}^{\max }=100 \mathrm{~ms}$. In this respect, the video receiver accepts only in-sequence frames whose end-to-end delay does not exceed $D_{1}^{\max }$. Any other frame is dropped with no subsequent retransmission. Therefore, the associated set of requirements could be captured by the minimum bit-rate $B_{1}^{\min }$ and a target frame loss ratio of $L_{1}^{\max }=0.05$ similarly to the work of [8]. An approach to assess the perceived QoE during an actual video transmission will be presented in Section IV-D.

- Data (i.e., delay-tolerant) transfer associated with a loose requirement in terms of a minimum bit-rate $B_{2}^{\min }=1 \mathrm{Kbps}$.

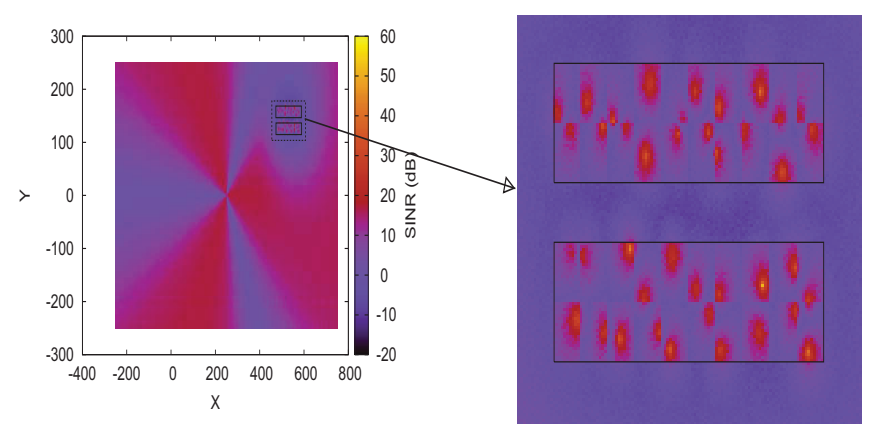

Fig. 3. Illustrative example of SINR map

In what follows, $A_{1}$ and $A_{2}$ will be used to denote the video and file transfer applications, respectively.

\section{Satisfaction levels}

- Interactive video: The generic model developed in Section III-A is instantiated for $R_{1}=2$ with $R e q_{1}^{1}=B_{1}^{\min }$ and $R e q_{1}^{2}=1 / L_{1}^{\max }$. Therefore, the satisfaction level associated with interactive video can be derived as:

$$
S L_{1, n, k}=\left(S L_{1, n, k}^{1}\right)^{\alpha_{1}^{1}} \cdot\left(S L_{1, n, k}^{2}\right)^{\alpha_{1}^{2}}
$$

where $S L_{1, n, k}^{1}$ and $S L_{1, n, k}^{2}$ denote the marginal satisfaction levels from the bit-rate and frame loss rate perspectives, respectively given by:

$$
\begin{gathered}
S L_{1, n, k}^{1}=\frac{\left(\frac{B_{1, n, k}}{B_{1}^{\text {min }}}\right)^{\xi_{1}^{1}}}{1+\left(\frac{B_{1, n, k}}{B_{1}^{\text {min }}}\right)^{\xi_{1}^{1}}} \\
S L_{1, n, k}^{2}=\frac{\left(\frac{1 / L_{1, n, k}}{1 / L_{1}^{\text {max }}}\right)^{\xi_{1}^{2}}}{1+\left(\frac{1 / L_{1, n, k}}{1 / L_{1}^{\text {max }}}\right)^{\xi_{1}^{2}}=\frac{\left(\frac{L_{1}^{\max }}{L_{1, n, k}}\right)^{\xi_{1}^{2}}}{1+\left(\frac{L_{1}^{\text {max }}}{L_{1, n, k}}\right)^{\xi_{1}^{2}}}}
\end{gathered}
$$

where $L_{1, n, k}$ denotes the frame loss rate perceived by the $n$-th user when using $R A T_{k}$.

- Data transfer: The generic model developed in Section III-A is instantiated for $R_{2}=1$ with $R e q_{2}^{1}=B_{2}^{\min }$. Therefore, the satisfaction level associated with file transfer can be derived as:

$$
S L_{2, n, k}=\left(S L_{2, n, k}^{1}\right)^{\alpha_{2}^{1}}=\left(\frac{\left(\frac{B_{2, n, k}}{B_{2}^{m i n}}\right)^{\xi_{2}^{1}}}{1+\left(\frac{B_{2, n, k}}{B_{2}^{m i n}}\right)^{\xi_{2}^{1}}}\right)^{\alpha_{2}^{1}}
$$

\section{Evaluation of video $Q o E$}

This section proposes an approach to evaluate the QoE perceived during interactive video sessions.

Given the high cost incurred by subjective tests, it is proposed to perform an objective assessment of the video QoE. In this respect, the Evalvid framework [9], whose simplified architecture is described in Fig. 4, has been integrated.

On the sender side, a digital video sequence is encoded, packetised and transmitted over a simulated network. On the receiver side, a play-out buffer is optionally used for jitter reduction before the received sequence is decoded and displayed. Both the sender and receiver keep track of the time-stamp and type of each sent/received packet in separate 


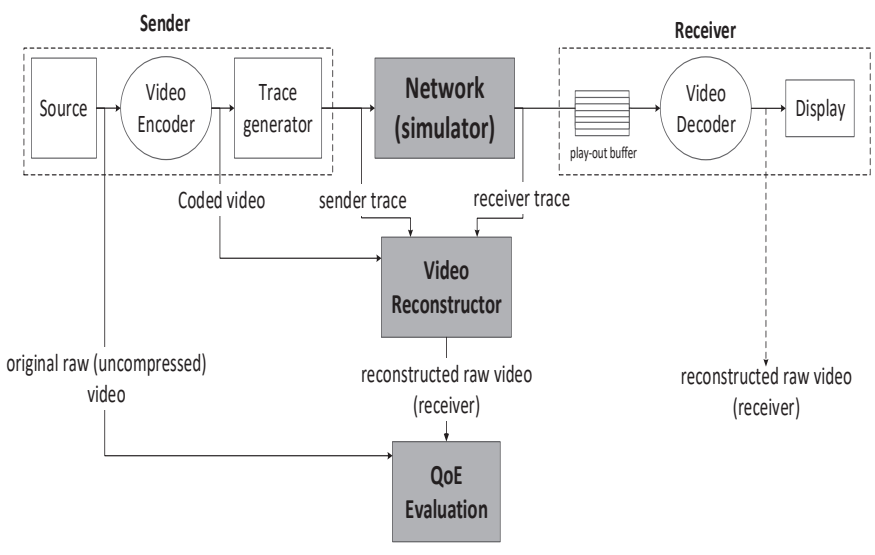

Fig. 4. A simplified architecture of the Evalvid framework

trace files. These trace files are combined with the original encoded video to reconstruct the uncompressed raw video as it would be perceived by the receiver. Based on a comparison between the original and reconstructed raw sequences, the QoE is evaluated based on the following metrics:

- The peak signal to noise ratio (PSNR) which has been extensively used in the literature for its simplicity. It provides a measure of the similarity between a distorted video sequence and its original counterpart based on a frame-by-frame comparison [9].

- The structural similarity (SSIM) index which focuses on the structural information loss to which the human visual system is strongly sensitive. It computes the mean, variance and covariance of small patches inside each frame and combines them into a distortion map [10].

To reflect the current trend in high-end video entertainment, the popular 10-min Big Buck Bunny animated video (e.g., 16+ million views on Youtube) has been selected as test sequence. Its uncompressed raw stream was downloaded from [11] and encoded with H264 (Main Profile, L4.1) at 1080p @24fps, which generates a variable bit-rate stream whose minimum requirement is $B_{1}^{\min }=10 \mathrm{Mbps}$.

\section{Performance evaluation}

To get insight into the effectiveness of the proposed metric, a set of system-level simulations have been performed using the NS-3 simulator [12].

\section{A. Initial assumptions}

A preliminary study has been conducted under the following assumptions:

- A single $10 m \times 10 m$ room of the dual-stripe layout described in Section. IV-A is initially considered. Therefore, the initial deployment area is $A=100 \mathrm{~m}^{2}$.

- The LTE macro and small cells operate on a bandwidth of $W=20 \mathrm{MHz}$. The associated medium access control (MAC) scheduling is performed based on the proportional fair (PF) algorithm [13].

- Inside the considered room, $N_{1}=1$ video session is established together with $N_{2} \in\{0,2,4\}$ file transfers during a simulation time of $T_{\text {sim }}=650 \mathrm{~s}$.

- The satisfaction levels of the video and file transfer applications are evaluated at $\xi_{1}^{1}=10, \xi_{1}^{2}=\xi_{2}^{1}=5$ and $\alpha_{m}^{r}=1$.

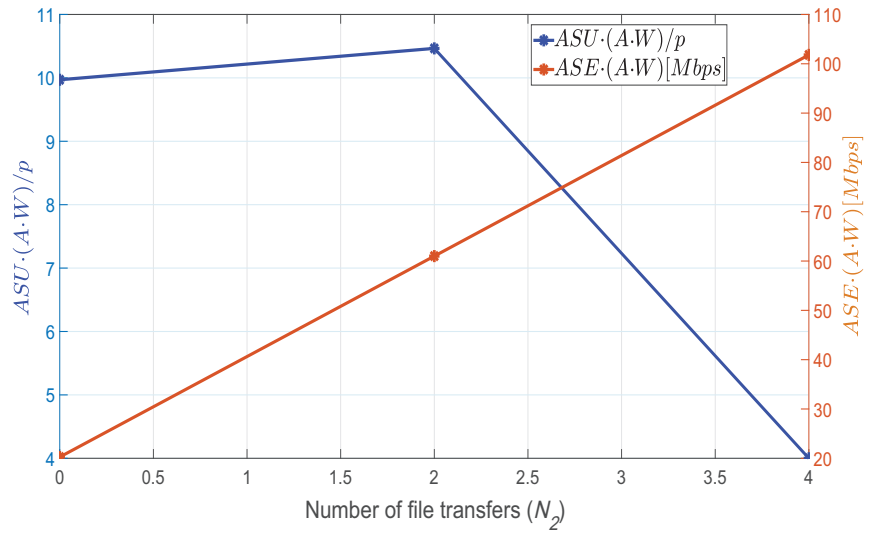

Fig. 5. Behavior of $A S U$ and $A S E$ for different traffic mixtures

- The pricing model of (6) is evaluated at $\beta=5$. To reflect the large share of revenue currently generated by video traffic, the maximum price $p_{m, n, k}$ associated with data transfer and video is set to $p$ and $10 p$, respectively.

\section{B. Initial results}

This section evaluates the ASU metric proposed in Section III-B and benchmarks it against the traditional $A S E$ defined in Section II-B for the considered use case in Section IV. The individual performances of each of the considered applications (i.e., interactive video and file transfer) are also evaluated together with their contributions to the overall $A S U$.

Fig. 5 plots the time average of both metrics when an increasing number of file transfers $\left(N_{2}\right)$ is established together with $N_{1}=1$ video session. For an intuitive interpretation of the obtained results, both $A S U$ and $A S E$ are multiplied by the area-bandwidth product to represent the aggregated revenue in multiples of the unit price $p$ (i.e., $A S U \cdot(A \cdot W) / p$ ) and bit-rate in Mbps (i.e., $A S E \cdot(A \cdot W)$ ), respectively.

The first key observation is that unlike $A S E, A S U$ first increases slightly at low loads of file transfers (i.e., $N_{2} \leq 2$ ), and then decreases sharply at higher loads. This is because, on the one hand, accepting more file transfers allows to consume a larger fraction of network resources. Thus, ASE increases linearly as it simply aggregates the bit-rate achieved by all users. On the other hand, $A S U$ values spectrum usage depending on the revenue generated by the established applications. When only few file transfers are active, the cross-traffic effects are minor, which justifies the slight increase of $A S U$ due to the contribution of these sessions. When the load increases further, the video traffic gets overwhelmed as per the PF scheduling, which strongly degrades its performance and significantly reduces the revenue it generates. As a result, $A S U$ is strongly impacted as the contribution of video users in (7) is much higher (i.e., $10 p$ ) compared to that of file transfers (i.e., $p$ ).

To understand better the observed behavior of $A S U$, the individual contributions of the considered applications are next analysed. Fig. 6(a) shows the average satisfaction levels achieved by the video and file transfer applications.

The results confirm that the file transfers associated with loose bit-rate requirements are fully satisfied even at the highest loads. In turn, the satisfaction level of the video user strongly depends on the load at hand. When the number of file transfers is low, the video performance is slightly reduced, and 


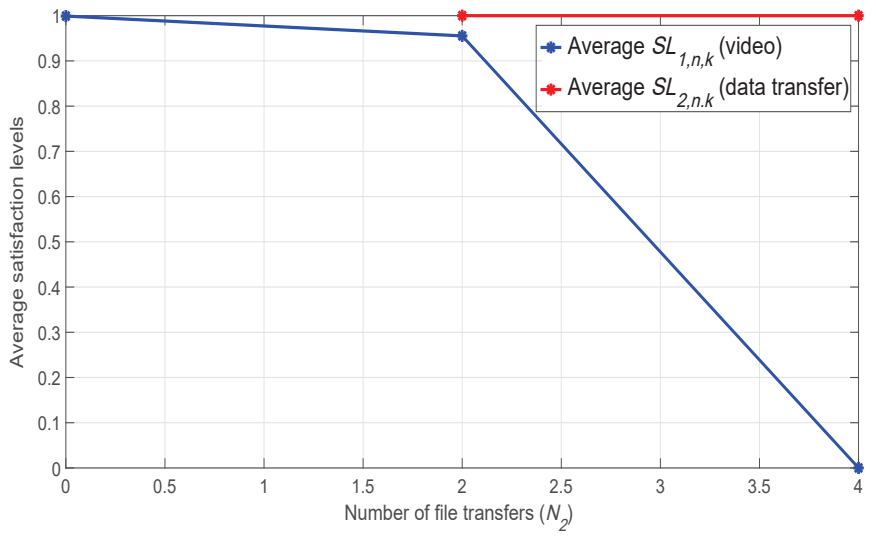

(a) Application satisfaction levels

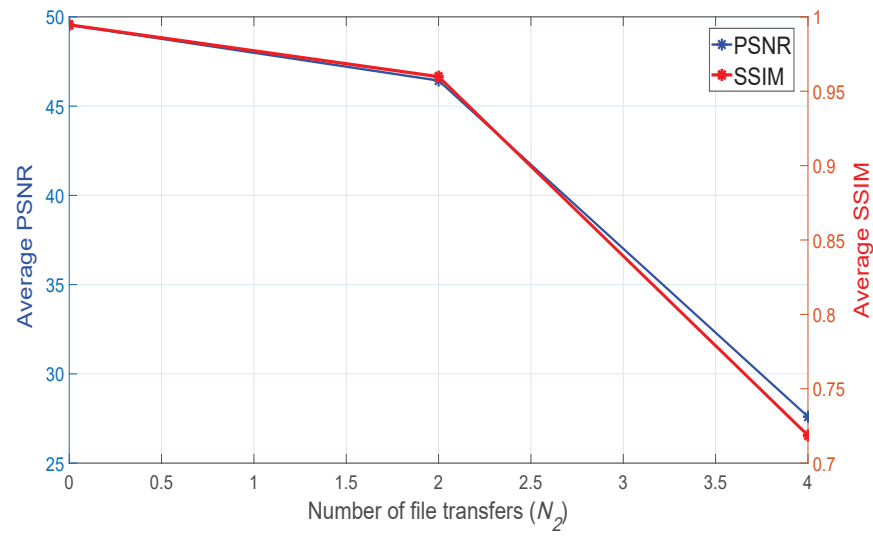

(b) Perceived QoE levels during video sessions

Fig. 6. Individual performances of the considered applications

so is its contribution to the total revenue in (7). However, the revenue brought by each new file transfer (i.e. $p$ ) dominates that reduction, and thus the overall $A S U$ slightly increases as previously observed in Fig. 5. As the load increases, the video application is strongly degraded and stops generating any revenue. This is clearly observed in Fig. 5, where it can be seen that $A S U$ equals exactly the contribution of file transfers (i.e., $4 p$ ) at the highest load (i.e., $N_{2}=4$ ). This calls for an admission control based on $A S U$ as rejecting file transfer requests at $N_{2}=2$ would be more beneficial from the total revenue perspective.

Finally, the performance of the video application is analysed in terms of the QoE metrics defined in Section IV-D. Fig. 6(b) shows the average PSNR and SSIM levels perceived during video sessions. It can be seen that when the file transfers overwhelm the video traffic, the QoE perceived by the enduser is strongly degraded particularly at the highest loads. This confirms that the satisfaction level formulation developed in Section IV-C captures well the end-user perception during video sessions.

In summary, the proposed $A S U$ significantly outperforms the traditional $A S E$ in assessing how efficiently spectrum resources are utilised from the total revenue perspective, particularly when the video QoE is degraded.

\section{CONCLUSIONS AND FUTURE WORK}

This paper proposes a novel spectrum utility (SU) metric that assesses the efficiency of spectrum usage by a set of heterogeneous applications. Unlike the traditional spectrum efficiency (SE), the proposed metric does not blindly consider the achievable bit-rate, but captures the most relevant performance metrics for each of the considered applications. In this respect, a generic formulation is first devised to assess the satisfaction level with respect to the various requirements. Then, the SU is formulated as an aggregated utility that combines these satisfaction levels with an innovative pricing model to derive the total revenue generated for the spectrum owner. To get insight into the usefulness of the proposed metric, the proposed methodology is instantiated for an illustrative use case, where a mixture of delay-sensitive (i.e., interactive video) and -tolerant (i.e., file transfer) applications are established in dense indoor deployments. The obtained results reveal that the proposed SU significantly outperforms the traditional SE in assessing how efficiently a limited frequency spectrum is utilised from the overall revenue perspective, particularly when the video quality-of-experience (QoE) is degraded. This calls for a novel SU-aware ecosystem, where the spectrum sharing models, billing policies and resource allocation mechanisms (e.g., medium access control (MAC) and radio resource management $(\mathrm{RRM})$ ) are jointly revisited to maximise the overall SU.

As part of future work, it is intended to extend the proposed SU metric to include the cost incurred on the network side, and construct a novel SU-aware framework, where the various degrees of freedom are jointly exploited.

\section{ACKNOWLEDGEMENTS}

The authors would like to gratefully acknowledge the support of BT through the University of Surrey 5G Innovation Centre (http://www.surrey.ac.uk/5gic), and the EU funded H2020 5G-PPP project SPEED-5G under the grant agreement no. 671705 .

\section{REFERENCES}

[1] W. C. Y. Lee, "Spectrum efficiency in cellular [radio]," IEEE Transactions on Vehicular Technology, vol. 38, no. 2, pp. 69-75, May 1989.

[2] M. S. Alouini and A. J. Goldsmith, "Area spectral efficiency of cellular mobile radio systems," IEEE Transactions on Vehicular Technology, vol. 48, no. 4, pp. 1047-1066, Jul 1999.

[3] "Evolved Universal Terrestrial Radio Access E-UTRA); Further advancements for E-UTRA physical layer aspects," 3GPP, Tech. Rep. 3GPP 36.814 v9.2.0, March 2017.

[4] L. Badia, M. Lindstrom, J. Zander, and M. Zorzi, "Demand and pricing effects on the radio resource allocation of multimedia communication systems," in GLOBECOM '03. IEEE, vol. 7, Dec 2003, pp. 4116-4121.

[5] "Simulation assumptions and parameters for FDD HeNB RF requirements," 3GPP, Tech. Rep. 3GPP R4-092042, May 2009.

[6] "NS-3 Model Library", Tech. Rep. Release NS-3.27, October 2017. [Online]. Available: https://www.nsnam.org/docs/release/3.27/models/ ns-3-model-library.pdf

[7] 3GPP, "Study on licensed-assisted access to unlicensed spectrum, (Release 12)," Tech. Rep. 36.889-V13.0.0, June 2015.

[8] W. Y. Lee and I. F. Akyldiz, "A spectrum decision framework for cognitive radio networks," IEEE Transactions on Mobile Computing, vol. 10, no. 2, pp. 161-174, Feb 2011

[9] J. Klaue, B. Rathke, and A. Wolisz, EvalVid - A Framework for Video Transmission and Quality Evaluation. Springer Berlin Heidelberg, 2003, pp. 255-272.

[10] Z. Wang, A. C. Bovik, H. R. Sheikh, and E. P. Simoncelli, "Image quality assessment: from error visibility to structural similarity," IEEE Transactions on Image Processing, vol. 13, no. 4, pp. 600-612, April 2004.

[11] The Big Buck Bunny. [Online]. Available: https://peach.blender.org/

[12] The network simulator-3 (NS-3). [Online]. Available: https://www. nsnam.org/

[13] R. Kwan, C. Leung, and J. Zhang, "Proportional fair multiuser scheduling in LTE," IEEE Signal Processing Letters, vol. 16, no. 6, pp. 461-464, June 2009. 